\title{
QUANTITATIVE ASSESSMENT OF ASTHMA SEVERITY IN PEDIATRIC EMERGENCY DEPARTMENT (ED) PATIENTS USING NONINVASIVE RESPIRATORY FUNCTION TESTS
}

\author{
M.E. Rodriguez ${ }^{1}$, K. Giordano ${ }^{2}$, M. Armani ${ }^{1}$, J. Richards ${ }^{3}$, M. Attia ${ }^{2}$, T.H. Shaffer ${ }^{1}$ \\ ${ }^{l}$ Nemours Research Lung Center / Biomedical Research, ${ }^{2}$ Emergency Medicine, ${ }^{3}$ Respiratory Care, A.I. \\ duPont Hosp for Children / Nemours, Wilmington, DE, USA
}

Background: We hypothesized that thoraco-abdominal synchrony (TAA) will correlate with impulse oscillation (IOS) markers of lung mechanics in symptomatic asthmatic childred.

Aims: To assess asthmatic severity scores in children and their correlation with IOS and respiratory inductance plethysmography (RIP) test results.

Methods: After IRB approval, we studied 40 children (3 to $18 \mathrm{yrs}$ ) with acute asthma in a pediatric ED. Following a physical examination, clinical asthma score and parental consent approval, measurements of TAA using RIP followed by IOS measurements of respiratory resistance (5-35 Hz). IOS severity score was assessed based on both Rrs5 and Xrs5 [zero (normal) to IV (very severe)].

Results: 22(55\%) children were compliant with IOS measurements (CV=5\%). Preschool-aged children cooperated with the technique as follows: 3 -yrs-old (50\%), 4-yrs-old (16\%), and 5-yrs-old (50\%). Most children, 38(95\%) were able to complete RIP measurements; $25(62 \%)$ exhibited phase angles $>$ than $25^{\circ}$. For IOS, 15(37.5\%) children, had abnormal lung function according to Rrs5 ( $>150 \%$ of predicted) and Xrs5 $\left(>1.5 \mathrm{cmH}_{2} \mathrm{O} / \mathrm{L} / \mathrm{s}\right.$ difference). The IOS severity scores were mild in 3 children, moderate in 7 , and severe in 5. The IOS severity score values correlated significantly $(\mathrm{p}=0.01)$ with markers of TAA (Phase Angle).

Conclusions: This study demonstrates the feasibility of obtaining ED measurements of respiratory resistance and inductance in acutely ill, asthmatic children. The IOS scoring system and RIP techniques are accurate methods to assess lung function in children with a wide range of asthma severity based on the clinical asthma score. 